\title{
Classification of Normal, Benign and Malignant Tissues using Fuzzy Texton and Support Vector Machine in Mammographic Images
}

\author{
Venkata Ragha Deepthi Loka \\ M.Tech Scholar, C.S.E \\ Vasireddy Venkatadri Institute of Technology \\ Nambur, Guntur, A.P, India
}

\author{
Sudhakar Putheti \\ Computer Science and Engineering \\ Vasireddy Venkatadri Institute of Technology \\ Nambur, Guntur, A.P, India
}

\begin{abstract}
Content Based Image Retrieval systems are helpful to the radiologists in diagnosis of breast cancer. This paper presents a method for retrieving breast tissue as normal, benign or malignant in mammograms by using FuzzyTextons. In feature extraction first fuzzy texton images of mammograms are calculated. During the detection of fuzzy texton, fuzzy based quantization is performed to get more accurate textons. Then feature vectors are extracted for fuzzy textons and for efficient classification and retrieval Support Vector Machine is used. The proposed method was tested for a mammogram set from MIAS database.
\end{abstract}

\section{General Terms}

Content based Image Retrieval

\section{Keywords}

Breast cancer, FuzzyTextons, Mammogram, SVM

\section{INTRODUCTION}

The analysis of medical images is important because medical image data has been increasing rapidly in hospitals and health care centers for performing clinical studies in digital form. For accessing and searching medical image databases is difficult. Therefore an appropriate information system has to be developed to efficiently retrieve and manage the collection of medical image data especially in the field of digital mammography.

Breast cancer is a type of cancer causing deaths in females around worldwide. It is examined that between one in eight and one in twelve women will develop breast cancer during their lifetime and one in thirty will die because of cancer[1]. The breast mass may be benign or malignant. Generally malignant tumor leads to cancer causing cells of the breast. A malignant tumor is a group of cancer cells that affects surrounding tissues and also spread to other parts of the body.

With the use of Mammography, breast cancer can be detected very effectively. But mammogram reading still remains a difficult clinical task since some breast cancers may produce changes in mammograms that are difficult to recognize [2]. During routine screening of mammograms it has been reported that $10-30 \%$ of lesions are misinterpreted [3] and also radiologists cannot accurately detect all breast cancers visible on mammograms. Therefore to improve detection accuracy and to reduce such variability and misinterpretation, content based image retrieval (CBIR) and computer-aided diagnosis (CAD) systems have been proposed.

CAD systems aid as a second opinion for radiologists in detecting and decision making processes and the variability reduction among radiologists interpretation of mammograms [4], [5].

Similar to CAD, CBIR systems use information extracted from images to represent them. In Content Based Image Retrieval system the images can be retrieved based on the visual features such as shape, color and texture [6]. In Content Based Image Retrieval System the user describes an image in terms of its visual features and the system retrieves similar images according to the query of the user. A CBIR system gives similar images according to a certain pattern without providing any diagnostic information related to the retrieved images. CBIR systems can aid diagnosis by providing efficient manipulation and organization of stored images. Hence the radiologist may use this CBIR in the detection of a lesion and its classification.

For image retrieval of mammograms, textural analysis based on fuzzy textons is to be studied. Texture is a global feature of CBIR that is widely used in medical images. Textural analysis can discover the texture signature of a medical image significant to the diagnostic problem. The effectiveness of textural analysis is determined by the methods used to extract meaningful features [7].

The proposed work initiates texture based analysis focusing on fuzzy textons which is a novel method to describe the texture features in low-dimensional feature spaces. This method builds on the definition of textons and SVM is used for the retrieval of the mammographies.

A number of research groups have been developing computer programs for analysis of mammography abnormalities.

Wolfe [8] proposed a classification scheme for mammograms based on the appearance of the parenchyma.

Campos et al, [9] used independent component analysis (ICA) and neural network multilayer perceptron to classify mammograms as normal, benign and malignant, with $98.7 \%$ of successful.

Honda et al. [10] developed a CBIR system for mammograms for identifying breast lesions. In this method, to extract textural features of mammograms from 136 clinical cases, a spatial gray level dependence matrices is used. For the performance evaluation of the process a selected set of ROIs and whole images were used from these cases. Precisions for selected ROIs and whole images ranged from $22 \%$ to $100 \%$ and from $31 \%$ to $100 \%$ respectively.

Yoshida et al. [11], [12] developed a system which is based on the wavelet transform. The least asymmetric Daubechies' wavelets in combination with a difference image technique is 
used in this method. These methods successfully separated microcalcifications from normal background tissue with approximately $90 \%$.

El-Naqa et al. [13] proposed an incremental learning based relevance feedback approach, for mammogram retrieval. It requires use of Support vector machine for developing an online learning procedure for similarity learning. This was implemented on clustered microcalcification images. It is reported that the retrieval is of more effective by using this approach.

Alto et al. [14] proposed the use of texture, gradient and shape measures as indices for quantitative representation of breast masses in mammograms. They suggested that features that can give high accuracy in pattern classification experiments could also be used as efficient indices for CBIR.

The mammographic appearance within the breast area can be captured by using textons by Petroudi et al. [15].

The textural features are widely used for detecting cancer in mammograms has given good performance on the tests of mammographic images from MIAS Database with recognition accuracy of $88.23 \%$ in the detection of all kinds of abnormalities and $79.31 \%$ in classifying benign and malignant regions. [16].

The rest of this paper organized as follows. Section 2 shows materials and methods, and results are shown in Section 3. Section 4 states the conclusion of this work.

\section{MATERIAL AND METHODS}

The proposed method has two steps like feature analysis and image classification and retrieval. Feature analysis is discussed with the Gray Level Co-occurrence matrix and the image classification retrieval is with the Bayesian Neural Network method.

\subsection{Feature Analysis}

The breast lesion may cause a disorder in the homogeneity of tissues so it is better to examine the textural features of mammograms. Lot of valuable information is contained by the textures of digital images, for further research and application. Previously there were many researches has been done on feature analysis among them Gray Level Spatial Dependence or Co-occurrence matrix (GLCM), a statistical textural method, to analyze the textural features of mammograms. The GLCM method is compared with the proposed method and is described as following.

\subsubsection{Gray Level Spatial Dependence Matrix (GLCM) vs Fuzzy Textons Based Image Retrieval} The gray-level spatial dependence or co-occurrence matrix (GLCM) measures the texture of the image by considering the intensities or grayscale values of the image at different distances and directions. As the co-occurrence matrices are typically large and sparse, various metrics of the matrix are often taken in order to get a more useful set of features. Features generated using this technique is usually called Haralick features. Haralick et al. [21] evaluated second order statistics on the corresponding GLCM for further texture description matrices. Second order measures consider the relationship between two neighboring pixels in the original image. The texture calculations require a symmetrical matrix. For each spatial relationship a different co-occurrence matrix exists (above, next to, diagonal). It is observed that the cooccurrence matrix is symmetrical and also the total number of pairs of compared pixels is different due to the angular relationships. To overcome this, it is necessary to normalize the frequencies of the occurrence. However, the simple intensity information does not provide adequate information especially for analysis and characterization of many medical images. Thus a new texture descriptor called Fuzzy Textons approach has been developed. Liu et al. in [22] was the first to use the term texton co-occurrence matrix.

For analyzing the textural features of mammograms and develops descriptors for content-based image retrieval, Fuzzy Textons, a statistical textural method is employed in this study. The proposed method is given below.

The word "Texton" is proposed by Julesz. A texton can be defined as a pattern sharing a common property all over the image [18]. Textures are formed only if the adjacent elements lie within the neighborhood Texton gradient depends on the critical distances between texture elements. Critical distance depends on the texture element size. Texture can be decomposed into elementary units, texton classes of colors, elongated blobs of specific width, orientation, aspect ratios and terminators of elongated blobs. If texture elements are greatly expanded in one orientation, discrimination reduces. If the elongated elements are not jittered in orientation, texture gradients increase at boundaries. Thus with a small sub image of size $3 \times 3$ is used to obtain texton gradient. The proposed method used 12 textons of $3 \times 3$ grids. If three pixels are highlighted and have same value then, grid will form a texton. Even the co-occurrence probability of same valued pixels in 3 $\mathrm{x} 3$ grid is smaller than that of $2 \times 2$ grid, the textons developed using $2 \times 2$ grid may not give complete information regarding direction. The computational complexity for fusing the overlapped components of 12 textons is also less to obtain final texton image. During the detection of fuzzy textons, fuzzy based quantization is performed to get more accurate textons as given in [28].

After the extraction of fuzzy texton images the texture features are extracted from them. Some of the important features of texture properties (cluster properties) used are Local Homogeneity, Cluster Shade and Cluster Prominence.

Local Homogenity: $\sum_{\mathrm{i}, \mathrm{j}=0}\left(1 /(\mathrm{i}-\mathrm{j})^{2}\right) \mathrm{C}(\mathrm{i}, \mathrm{j})$

Cluster Shade: $\sum_{\mathrm{i}, \mathrm{j}=0}\left(\mathrm{i}-\mathrm{M}_{\mathrm{x}}+\mathrm{j}-\mathrm{M}_{\mathrm{y}}\right)^{3} \mathrm{C}(\mathrm{i}, \mathrm{j})$

Cluster Prominence: $\sum_{\mathrm{i} . \mathrm{j}=0}\left(\mathrm{i}-\mathrm{M}_{\mathrm{x}}+\mathrm{j}-\mathrm{M}_{\mathrm{y}}\right)^{4} \mathrm{C}(\mathrm{i}, \mathrm{j})$

Where $\mathrm{M}_{\mathrm{x}}=\sum_{\mathrm{i}, \mathrm{j}=0}$ i C(i,j);

$M_{y}=\sum^{n_{i . j}=0}$ j C $(i, j)$

\subsection{Image Retrieval}

The Objective of the CBIR system is to examine the textural features of mammograms and to discriminate malignant from benign and normal tissues

\subsubsection{Bayesian Neural Networks vs Support Vector Machine}

In the Bayesian approach to learning neural network [23], [24], [25], the objective is to find the weights posterior distribution mode. To obtain the posterior distribution of the weights, first the prior distribution is specified, which is a probability distribution that represents the prior information associated with the weights of the network, and the data likelihood. Once the prior distributions have chosen, the evidence from the data is combined to get the posterior distribution for the parameters and hyperparameters. In a multi-class classification problem, the targets are represented 
by a binary label vector so that a single component is set to one to denote the correct class and the rest of components are set to zero. In the Bayesian learning, the posterior distribution is used to find the predictive distributions for the target values in the new test case given the inputs for that case as well as inputs and target for training cases [26].

In Bayesian Neural Network specifying a prior is extremely difficult. Even though the prior is specified computing a posterior may be extremely difficult such that computational approximation is required.

The proposed method used Support Vector Machine instead of Bayesian Neural Networks as the support Vector Machine has more generalization power than Bayesian Neural Network Classifier [27]. This method is given below.

In this paper SVM classifier is used for image retrieval. SVM was used to indicate the relevance of the images to a certain query. The support vector machine (SVM) method was developed for classification problems [19] and also for retrieval systems [20].

The SVM constructs a hyperplane in the feature space which can be used for classification. It separates the points with the highest number of points of the same class on the same side, while maximizing the distance between two parallel hyperplanes. The hyperplane constructed can be determined by using subset of items from the two classes which are called as support vectors. If the set of data is separated into two classes by a straight line it is called a linear classification. For nonlinear classification SVM kernel is used.

If data set has more than two classes it becomes a multi-class problem. One approach to solve the multi-class problems is by reducing the single multiclass problem into set of binary problems, using methods of decomposition one by class (one against all) and the separation of every pair of classes (one against one). In the one-against-all method, a SVM is built for each class through the discrimination of this class against the remaining classes. The number of SVMs used in this method is $\mathrm{N}$. Test data $\mathrm{x}$ are classified using a decision strategy, i.e., the class with the maximum value for the discriminate function $f(x)$ is assigned to the data. The $n$ training examples are used to construct the SVM for one class. The SVM for one class $\mathrm{p}$ is built using the set of training data (x) and the desired outputs (y).

In the one-against-one method, a SVM is built for a pair of classes through training, for the discrimination of two classes. In this way, the number of SVMs used in the method is $\mathrm{N}(\mathrm{N}-1) / 2$. One SVM for a pair of classes is built using training examples belonging only to these classes. The other approach is a generalization of the binary classification to more than two classes. This paper uses the one-against-one method. A 10-fold cross validation was performed and the tests were conducted using the polynomial kernel.

\section{EXPERIMENTAL RESULTS}

As the Mini MIAS database consists of 332 mammograms of different categories, it has been selected for the testing of performance of the proposed algorithms. The regions of interest (ROI) were manually extracted from each image based on the information provided by the MIAS database. To each mammogram, a ROI was manually selected, containing the lesion. If the tissues had different sizes, ROIs was resized to $256 \times 256$ pixels. Here benign, malignant and normal tissues were considered deliberately as shown in figure 1 .

\subsection{Mammogram Database}

The Proposed method used the Mammographic Institute Society Analysis mini-MIAS [17] database. This database consists of 332 mammograms of right and left breast, from 161 patients, where 53 were diagnosed as being malignant, 69 benign and 206 normal with ages ranging from 50 to 65 . The spatial resolution of the image is $50 \mu \mathrm{m} \times 50 \mu \mathrm{m}$. The images are digitized at a resolution of $1024 \times 1024$ pixels and at 8-bit grey scale level and these also include the locations of all abnormalities that may be present. The existing data in the collection consists of the location of the abnormality (like the center of a circle surrounding the tumor), its radius, breast position (left or right), type of breast tissues (fatty, fattyglandular and dense) and tumor type if it exists (benign or malignant).

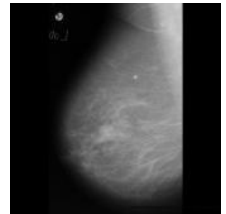

(a)

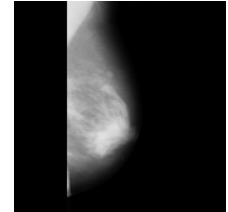

(b)

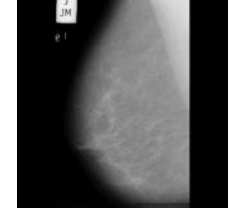

(c)
Figure 1: Mammographies of different breast tissues: (a) Benign, (b) Malignant, (c) Normal.

\subsection{Results}

The textural features were extracted as mentioned in section 2.1 and compared to the features in the MIAS reference database using the SVM method. A mean success rate of $90 \%$ on discriminating malignant from benign and normal tissues was achieved by this proposed method that was shown in Table 1.

Table 1. Accuracy analysis of Breast tissue characterization

\begin{tabular}{|c|c|c|c|}
\hline Tissues & Benign & Malignant & Normal \\
\hline Total & 13 & 16 & 31 \\
\hline Correct & 9 & 15 & 30 \\
\hline Correct \% & 69.2 & 93.8 & 96.8 \\
\hline
\end{tabular}

The algorithm achieved good classification compared to Co-occurrence matrix and Bayesian Neural Network.

\section{CONCLUSION}

In this paper we presented a CBIR system for retrieving tissues in mammograms as benign, malignant or normal and is able to aid radiologists in their diagnosis. In this method we used fuzzy textons which is a novel method to describe the texture features in low-dimensional feature spaces. This method builds on the definition of textons. Hence the proposed classification technique assists the radiologists in their diagnosis. The method employed here has given better performance. 


\section{REFERENCES}

[1] N. C. I. (NCI). Cancer stat fact sheets: Cancer of the breast, 2006 .Available at http://seer.cancer.gov/statfacts/html/breast.html.

[2] Strickland, R.N. and H.L. Hahn, 1996. Wavelet transforms for detecting microcalcifications in mammograms. IEEE Trans. Med. Imag., 15:218-119.

[3] Kopans, D.B., 1992. The positive predictive value of mammography. Am.J.Roentgenol, 158:521-526.

[4] Chan H P, Doi K, Vybamy C J, Schmidt R A, Metz C E, Lam K L, Ogura T, Wu Y and MacMahon H 1989 Improvement of radiologists' detection of clustered microcalcifications on mammograms: the potential of computer-aided diagnosis Invest. Radiol. 25 1102-10

[5] Y. Jiang, R. M. Nishikawa, R. A. Schmidt, A. Y. Toledano, and K. Doi. Potential of computer-aided diagnosis to reduce variability in radiologists interpretations of mammograms depicting microcalcifications. Radiology, 220:787-794, 2001.

[6] Rubner, Y. and C. Tomasi, Perceptual Metrics for Image Database Navigation. The Kluwer International Series in Engineering and Computer Science. 2001: Kluwer Academic Publishers. 137.

[7] G. D. Tourassi, "Journey toward computer-aided diagnosis: role of image texture analysis," Radiology, 213(2), pp.317-320, 1999

[8] J.N. Wolfe. Risk for breast cancer development determined by mammographic parenchymal pattern. Cancer, 37:2486-2492, 1976.

[9] Campos, L. F. A. ; Barros, A. K. ; Silva, A. C. "Independent Component Analysis and Neural Networks Applied for Classification of Malignant, Benign and Normal Tissue in Digital Mammography", In: Special Issue - Methods of Information in Medicine, v. 46, p. 212-215, 2007

[10] M. O. Honda, P. M. A. Marques, and J. A. H. Rodrigues, "Content-based image retrieval in mammography: using texture features for correlation with BI-RADS categories," Proceedings of the 6th International Workshop on Digital Mammography, pp. 401-403, 2002.

[11] H. Yoshida, R. Nishikawa, K. Muto, K. Doi, and M. Tsuda, "Application of the wavelet transform to automated detection of clustered microcalcifications in digital mammograms," Tokyo Inst. Polytech., Tokyo, Japan, Academic Rep., vol. 16, 1994.

[12] H. Yoshida, R. Nishikawa, G. Maryellen, and K. Doi, "Computer-aided diagnosis in mammography: Detection of clustered microcalcifications based on multiscale edge representation," in Computer Assisted Radiology. Amsterdam, The Netherlands: Elsevier, 1996.

[13] El-Naqa I, Y. Yang, N. P. Galatsanos, and M. N. Wernick, "Relevance feedback based on incremental learning for mammogram retrieval," Proceedings of the International Conference on Image Processing 2003, pp.729-732, 2003.
[14] Alto H, Rangayyan RM, Desautels JEL: Content-based retrieval and analysis of mammographic masses. J Electron Imaging 14(2): Article 023016:1Y17, 2005

[15] S. Petroudi, T. Kadir, and M. Brady, "Automatic classification of mammographic parenchymal patterns: A statistical approach," in Proc. IEEE Conf. Eng. Med. Biol. Soc., 2003, vol. 1, pp. 798-801.

[16] A. K. Christoyianni, E. Dermatas, and G. Kokkinakis. Computer aided diagnosis of breast cancer in digitized mammograms. Computerized Medical Imaging and Graphics, 26:309-319, 2002.

[17] J. Suckling, J. Parker, D. Dance, S. Astley, I. Hutt, C. Boggis, and et al. The mammographic images analysis society digital mammogram database. Exerpta Medical, 1069:375-378, 1994

[18] B. Julesz. Texton gradients: The texton theory revisited. Biological Cybernetics, 54(1986) 245-251.

[19] M.F. Akay, Support vector machines combined with feature selection for breast cancer diagnosis, Expert Systems with Applications, 36 (2009)3240-3247.

[20] W.T.Wong, S.H. Hsu, Application of SVM and ANN for image retrieval, European Journal of Operational Research 173 (2006) 938-950.

[21] R. Haralick, K. Shanmugam, and I. Dinstein, "Textural features for image classification," Systems, Man and Cybernetics, IEEE Transactions on, vol. 3, pp. 610-621, 1973.

[22] G.-H. Liu and J.-Y. Yang, "Image retrieval based on the texton cooccurrence matrix," Pattern Recognition, vol. 41, no. 12, pp. $3521-3527,2008$.

[23] W. L. Buntine and A. S. Weigend. Bayesian backpropagation. Complex Systems, 5:603-643, 1991.

[24] D. J. C. Mackay. Bayesian Methods for Adaptive Models. Tese de doutorado, California Institute of Technology, 1991.

[25] D. J. C. Mackay. A practical Bayesian framework for backpropagation networks. Neural Computation, 4(3):448-472, 1992.

[26] M. Ghosh, T. Maiti, S. Chakraborty, and A. Tewari. Hierarchical bayesian neural networks: An application to prostate cancer study. Journal of the American Statistical Association, 99(467):601-608, 2004

[27] Geraldo Braz Junior, Leonardo De Oliveira Martins, Aristófanes Correa Silva, And Anselmo Cardoso Paiva. Comparison Of Support Vector Machines And Bayesian Neural Networks Performance For Breast Tissues Using Geostatistical Functions In Mammographic Images: International Journal of Computational Intelligence and Applications 2010 09:04, 271-288.

[28] CBIR using Texels of colour Fuzzy Textons S Putheti, SR Edara, SA Edara - Hybrid Intelligent Systems(HIS) 2012 IEEE $12^{\text {th }}$ International Conference. 